\title{
Initial academic experience and learning curve with robotic spine instrumentation
}

\author{
Timur M. Urakov, MD, Ken Hsuan-kan Chang, MD, S. Shelby Burks, MD, and Michael Y. Wang, MD \\ Department of Neurological Surgery, University of Miami Miller School of Medicine, Miami, Florida
}

OBJECTIVE Spine surgery is complex and involves various steps. Current robotic technology is mostly aimed at assisting with pedicle screw insertion. This report evaluates the feasibility of robot-assisted pedicle instrumentation in an academic environment with the involvement of residents and fellows.

METHODS The Renaissance Guidance System was used to plan and execute pedicle screw placement in open and percutaneous consecutive cases performed in the period of December 2015 to December 2016. The database was reviewed to assess the usability of the robot by neurosurgical trainees. Outcome measures included time per screw, fluoroscopy time, breached screws, and other complications. Screw placement was assessed in patients with postoperative CT studies. The speed of screw placement and fluoroscopy time were collected at the time of surgery by personnel affiliated with the robot's manufacturer. Complication and imaging data were reviewed retrospectively.

RESULTS A total of 306 pedicle screws were inserted in 30 patients with robot guidance. The average time for junior residents was $4.4 \mathrm{~min} / \mathrm{screw}$ and for senior residents and fellows, $4.02 \mathrm{~min} / \mathrm{screw}$ ( $p=0.61)$. Among the residents dedicated to spine surgery, the average speed was $3.84 \mathrm{~min} / \mathrm{screw}$, while nondedicated residents took $4.5 \mathrm{~min} / \mathrm{screw}(p=$ 0.41). Evaluation of breached screws revealed some of the pitfalls in using the robot.

CONCLUSIONS No significant difference regarding the speed of pedicle instrumentation was detected between the operators' years of experience or dedication to spine surgery, although more participants are required to investigate this completely. On the other hand, there was a trend toward improved efficiency with more cases performed. To the authors' knowledge, this is the first reported academic experience with robot-assisted spine instrumentation.

https://thejns.org/doi/abs/10.3171/2017.2.FOCUS175

KEY WORDS robotics; minimally invasive; pedicle screw; spinal fusion; image guidance; Mazor

$\mathrm{T}$ HORACOLUMBAR spinal fusion and instrumentation procedures are complicated, requiring the surgeon to have special expertise in the planning, technical, and anatomical features that may be unique to each case. For this reason, the education and training of a spinal surgeon, be it in the orthopedic or neurosurgical realm, is long and arduous. Only through years of practice is a surgeon able to competently and reliably engage in thoughtful preoperative planning, tissue dissection and exposure, neural decompression, bone grafting, and instrumentation. Thus, any technological aids to enhance patient safety and improve the acquisition of surgical skills would be most welcome.

By definition, a robot is a machine capable of automating one or a series of actions. Robotics in spine surgery has been gaining popularity. To date, the use of robots has been largely limited to assistance with pedicle screw placement. Through the merging of image-based data and surgical preplanning, robotics may allow the surgeon to place pedicle screws in a more reliable fashion, theoretically allowing the procedure to become more stereotyped and automated. ${ }^{1,3-9}$ This also allows the surgeon to deal 
with variations in patient anatomy, such as small atrophic pedicles or complex spinal deformity. Thus, the addition of a robot (or more accurately a "cobot") takes the procedure beyond a passive image guidance system, as the robot provides a platform for pedicle screw trajectories.

Interestingly, to date most of these robotic systems have not found use in the academic or residency training environment. Several explanations exist for this phenomenon: 1) The high capital equipment costs are prohibitive. 2) The large volume seen in academic centers might make usage of a single robot impractical for regular use when multiple surgeries are performed daily. 3) Academic surgeons might believe that their specialized surgical skills do not require this technological addition. 4) There is a perceived risk of trainees developing a reliance on this new technology. Thus, almost all published North American studies of robotic pedicle screw placement have involved a limited number of attending surgeons who have concentrated their learning curves and already demonstrated commitment or "buy in" with this new technology.

Our central research question is, in a real-world training environment where residents and fellows interact in complex systems, would the robot be helpful? To address this, in this report we explored the variability in speed and efficacy of pedicle screw placement between trainees at different levels of experience. For these technologies to disseminate widely, they would have to be usable and practical for trainees developing their surgical skills.

\section{Methods}

\section{Patient Selection}

We performed a retrospective review of prospectively collected data in 33 consecutive patients in whom robotassisted pedicle instrumentation was planned during the period of December 2015 to December 2016. Robot-related data were recorded prospectively and included demographics, surgeons involved, time of operation, fluoroscopy use, screws executed, and notes on intraoperative adverse events. Patient charts were reviewed retrospectively for operative reports, follow-up notes, and imaging. All surgeries were performed at the same hospital led by a single attending surgeon.

\section{Residents}

Residents and fellows were assigned to cases based on the daily operating room coverage requirements. None of the trainees had received prior training on the robot, such as cadaveric courses, instructional didactics, or continuing medical education-approved courses. All residents and fellows directly performed robot-assisted instrumentation under the attending surgeon's supervision with guidance provided as needed. Speed was determined based on the total time the robot was used, from the moment the robot was oriented for the first screw until the last screw was placed, divided by the number of pedicle screws placed in each case. For the purpose of data collection, resident experience and seniority was assigned based on years in training. Residents in their 6th year and above were considered seniors, while residents in postgraduate years (PGYs) 1-5 were considered juniors. All participating res- idents and fellows were asked about their career choices. Those stating spine surgery as their future specialty were assigned to "dedicated" group, while others to the "nondedicated" group.

\section{Robotic System and Operative Procedure}

The Renaissance Guidance System (Mazor Robotics) was used in all cases for pedicle instrumentation. Preoperative planning for pedicle screw trajectories was made using Mazor Robotics' proprietary software utilizing preoperative CT scans with 1-mm cuts. During surgery, once the mounting platform was secured to the patient, anteroposterior and oblique radiographs were obtained. Through these images, the software registered the platform to the preoperative plan. The robot was then attached to the platform and used to direct the instruments according to the preplanned trajectories. Time points were captured and recorded prospectively by Mazor software and representatives. The initiation of screw placement was based on when the robot moves to its first screw trajectory. Conclusion of screw placement was when then robot was removed and last screw seated. Fluoroscopy time was measured using the $\mathrm{C}$-arm machine, and representatives from Mazor Robotics recorded this information.

Open versus percutaneous screw placement was made on a case-by-case basis. All pedicle screws were manufactured by DePuy and used the Expedium Spine System or Viper System (DePuy Synthes) depending on whether placement was open or percutaneous.

\section{Breached Screw Assessment}

All available postoperative $\mathrm{CT}$ scans were reviewed for accuracy. Breached screws were assigned Grades 1,2, or 3 ( $<2 \mathrm{~mm}, 2-4 \mathrm{~mm},>4 \mathrm{~mm}$, respectively). The breached screws were then compared with the preoperative trajectory plan. The starting point location, tip location from the midline, and angle of the screw were measured.

\section{Statistical Analysis}

The 2-tailed Student t-test and Fisher's exact test were used to compare statistical groups.

\section{Results}

In the year between December 2015 and December 2016 , a total of 33 robot-assisted cases were planned. One case was canceled due to patient's deteriorated clinical condition before surgery. In one case the robot's use was canceled due to outdated software that was discovered before the case. In another case the robot was used to navigate toward a T-4 vertebral abscess for drainage without instrumentation. The remaining 30 patients had a total of 306 pedicle screws placed at the T4-pelvis levels. There were 15 females and 15 males with an average age of 70 years (range 46-85 years). Twenty-four surgeries were open ( 256 screws) and 6 were percutaneous (50 screws). The average time per screw insertion was $3.6 \mathrm{~min} / \mathrm{screw}$ in the open and $5.7 \mathrm{~min} / \mathrm{screw}$ in the percutaneous approaches $(\mathrm{p}=0.009)$. Fluoroscopy time was $11.1 \mathrm{sec} / \mathrm{case}$ and $1.15 \mathrm{sec} / \mathrm{screw}$ in the open cases and $11.8 \mathrm{sec} /$ case and 
TABLE 1. Summary of data for Renaissance Guidance System-assisted pedicle screw instrumentation

\begin{tabular}{|c|c|c|c|c|}
\hline Variable & Open & Percutaneous & Total & $\begin{array}{c}p \\
\text { Value }\end{array}$ \\
\hline No. of cases & 24 & 6 & 30 & \\
\hline Male & 14 & 1 & 15 & \\
\hline Female & 10 & 5 & 15 & \\
\hline Mean age (yrs) & 70 & 65.5 & & \\
\hline No. of pedicle screws inserted & 256 & 50 & 306 & \\
\hline Mean time (min/screw) & 3.6 & 5.7 & & $0.009^{*}$ \\
\hline $\begin{array}{l}\text { Mean fluoroscopy time } \\
\text { (sec/screw) }\end{array}$ & 1.15 & 1.73 & & 0.13 \\
\hline Revision surgery & 8 & 1 & 9 & \\
\hline Mean speed (min/screw) & & & & 0.158 \\
\hline Revision surgery & 3.8 & & & \\
\hline Initial surgery & 4.5 & & & \\
\hline $\begin{array}{l}\text { Mean fluoroscopy time } \\
\text { (sec/case) }\end{array}$ & & & & 0.059 \\
\hline Revision surgery & 7.4 & & & \\
\hline Initial surgery & 12 & & & \\
\hline \multicolumn{5}{|l|}{ Screw position on postop CT } \\
\hline $\begin{array}{l}\text { No. of screws available for } \\
\text { review }\end{array}$ & 77 & 24 & 101 & $0.033^{*}$ \\
\hline Breached & 6 & 6 & 12 & \\
\hline Grade 1 & 3 & 4 & 7 & \\
\hline Grade 2 & 3 & 1 & 4 & \\
\hline Grade 3 & 0 & 1 & 1 & \\
\hline
\end{tabular}

* Statistically significant.

$1.73 \mathrm{sec} / \mathrm{screw}$ in percutaneous cases on average $(\mathrm{p}=0.61$ [sec/case] and $\mathrm{p}=0.13$ [sec/screw]) (Table 1).

Of the 30 patients who underwent screw placement, 21 patients were undergoing their first implantation. The diagnosis included degenerative spine disease $(n=8)$, spinal deformity $(\mathrm{n}=12)$, and compression fracture with instability $(\mathrm{n}=1)$. Nine robot-assisted cases were revision surgeries for adjacent-level disease (4), proximal junctional
TABLE 2. Average speed of screw implantation based on level of experience and dedication to spine surgery

\begin{tabular}{lccc}
\hline \multicolumn{1}{c}{ Variable } & $\begin{array}{c}\text { No. of } \\
\text { Participants }\end{array}$ & $\begin{array}{c}\text { Speed } \\
\text { (min/screw) }\end{array}$ & $\begin{array}{c}\mathrm{p} \\
\text { Value }\end{array}$ \\
\hline Participant & 7 & 4.4 & 0.61 \\
\hline $\begin{array}{l}\text { Junior (PGYs 1-5) } \\
\text { Senior residents \& fellows } \\
\text { (PGYs 6-8) }\end{array}$ & 5 & 4.02 & \\
\hline Dedication to spine surgery & & & 0.41 \\
\hline Dedicated & 5 & 3.84 & \\
\hline Not dedicated & 7 & 4.5 & \\
\hline Speed & & & 0.057 \\
\hline 1st 3 cases & 25 & 4.6 & \\
\hline 4 cases & 9 & 3.6 & \\
\hline
\end{tabular}

kyphosis (4), and pseudarthrosis (1). Total fluoroscopy use in revision cases was $7.4 \mathrm{sec} /$ case versus $12 \mathrm{sec} /$ case in initial cases $(p=0.059)$. Only 2 of 9 revision cases required extra fluoroscopy due to additional work done (extreme lateral interbody fusion and pedicle subtraction osteotomy/discectomy) versus 11 of 20 initial cases. Revision cases required $3.8 \mathrm{~min} / \mathrm{screw}$ vs $4.5 \mathrm{~min} / \mathrm{screw}$ in initial cases $(\mathrm{p}=0.158)$.

\section{Residents and Fellows}

Twelve residents and fellows (PGYs 1-8) were involved in 1-7 robot-assisted procedures in which $8-70$ pedicle screws were inserted. The average time for junior residents (PGYs 1-5) was $4.4 \mathrm{~min} / \mathrm{screw}$ and for senior residents and fellows (PGYs 6-8) $4.02 \mathrm{~min} / \mathrm{screw}(\mathrm{p}=$ 0.61 ) (Fig. 1 and Table 2). Among the residents dedicated to spine surgery, the average speed was $3.84 \mathrm{~min} / \mathrm{screw}$, while nondedicated residents took $4.5 \mathrm{~min} / \mathrm{screw}$ ( $\mathrm{p}=$ 0.41) (Fig. 2). Dedication to spine surgery was assigned based on the current residents' career plans. The average

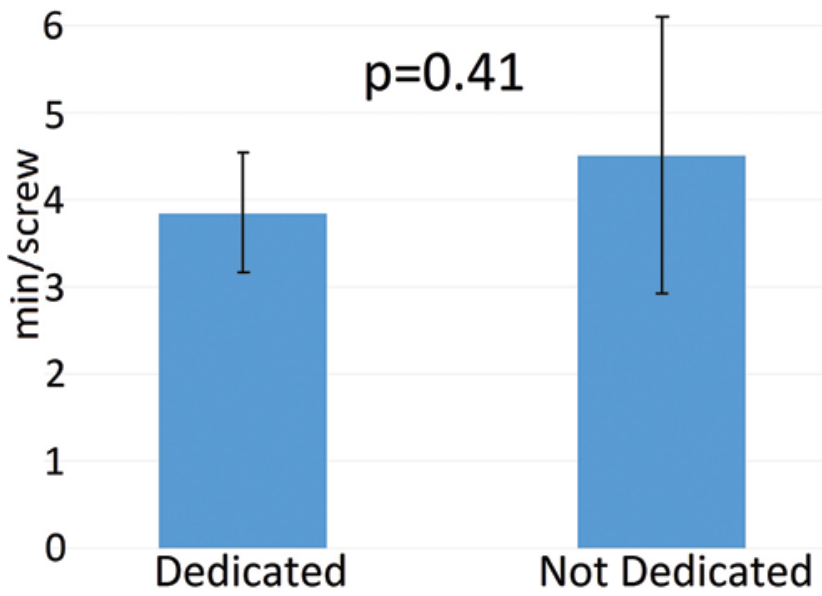

FIG. 2. Pedicle screw insertion speed based on residents' dedication to spine surgery.

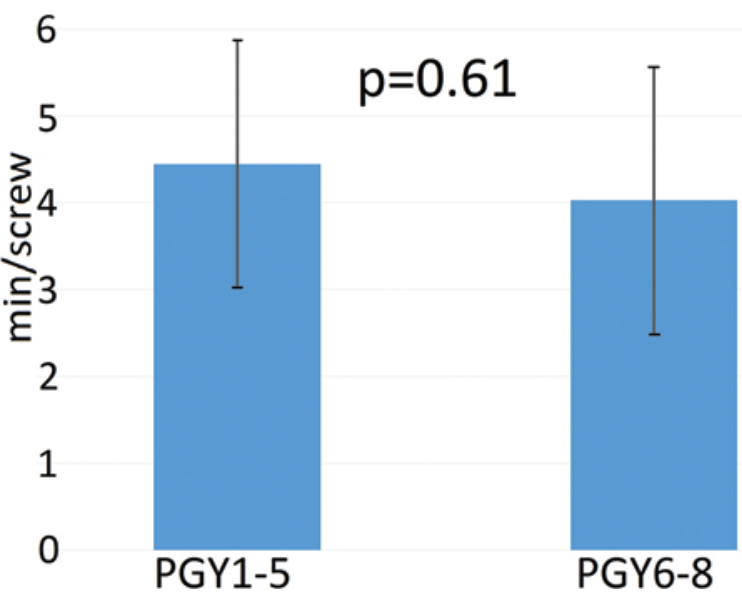

FIG. 1. Pedicle screw insertion speed based on years in training 

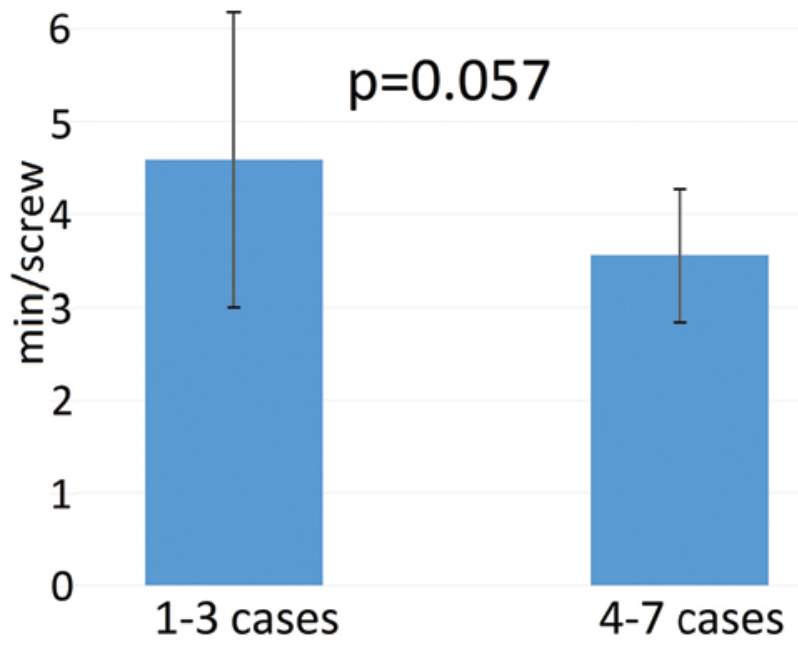

FIG. 3. Pedicle screw insertion speed in the first 3 cases versus subsequent cases.

time for residents in their first 3 surgeries was $4.6 \mathrm{~min} /$ screw versus $3.6 \mathrm{~min} / \mathrm{screw}$ in the subsequent robotic surgeries ( $p=0.057$ ) (Fig. 3). The fastest case recorded took $1.9 \mathrm{~min} / \mathrm{screw}$ in the 4-level open lumbar instrumentation and the slowest was $7.8 \mathrm{~min} / \mathrm{screw}$ by a PGY 7 resident on his first robotic percutaneous lumbar case (Fig. 4).

\section{Intraoperative Adverse Events}

The robot was unavailable in one case due to "timedout" software. In 1 case, bridge joints became loose, leading to 5 misplaced K-wires. Once the problem was recognized, the robot was reregistered and all final screws were in good position. In 2 cases, a K-wire was accidentally dislodged and required reinsertion. In 2 cases the drill guide skived inferiorly upon docking on the sloped bony surface, resulting in incorrect placement of the screws. This was recognized intraoperatively and screws were redirected in proper trajectory. In 2 open cases, percutaneous instruments had to be used at the lower levels because of inadequate soft-tissue retraction. No CSF leaks or changes in neuromonitoring findings were identified during any of the robot-assisted parts of the procedures.

\section{Spine Manipulation Before Robot Registration}

Thirteen cases required substantial manipulation of intervertebral space prior to instrumentation. Interbody graft from posterior $(n=4)$, anterior $(n=2)$, and lateral (n $=4)$ approaches, vertebroplasty $(n=1)$, thoracic pedicle subtraction for discectomy $(\mathrm{n}=1)$, and Ponte osteotomy $(n=1)$ was done before the robot registration with 132 robot-assisted screws placed after. There were no breached screws in this group. Robot registration required $5.2 \mathrm{sec}-$ onds of fluoroscopy in manipulated group versus $5.4 \mathrm{sec}-$ onds in patients without manipulations prior to registration $(\mathrm{p}=0.54)$.

\section{Breached Screws}

Nine patients had a postoperative CT study available

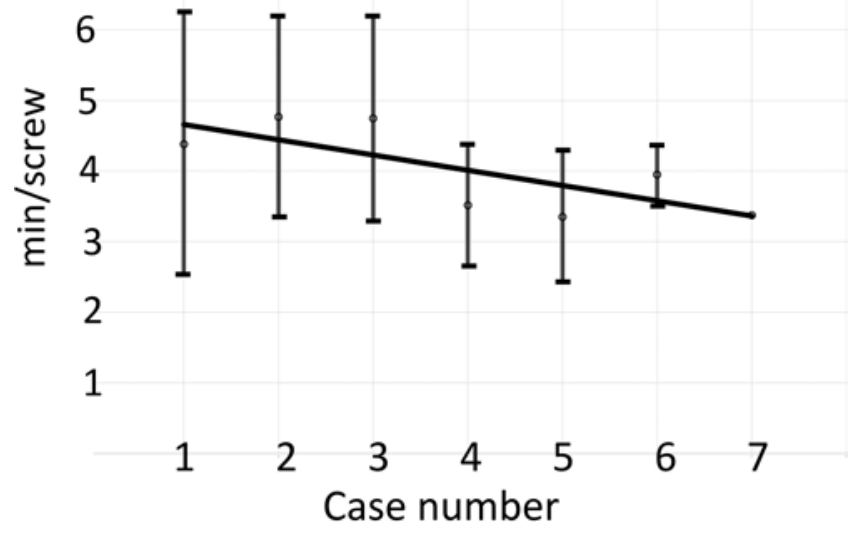

FIG. 4. Time per screw required by the residents with more cases performed. Points represent mean values and error bars are standard deviations. The thick line is the linear trend line.

for review with a total of 101 screws. In open cases, 6 of 77 screws were breached, while in percutaneous cases, 6 of 24 screws were breached $(\mathrm{p}=0.033)$ (Table 1). One screw had a Grade 3 breach laterally (L-5), 3 screws had a Grade 2 breach medially (T-10, L-1, and L-3), 1 screw had a Grade 2 breach superiorly (L-5), and 7 screws had less than a 2-mm breach. Four screws in the lower thoracic area (T10-L1) were breached due to a medial shift in the starting point. Four screws in the lower lumbar area (L-5, S-1) were breached due to lateral shift in the starting point (Fig. 5).

\section{Complications}

There was no CSF leakage or neuromonitoring changes with robot use. CSF leakage was encountered in 2 patients during the laminectomy portion of the cases and were repaired primarily with 4-0 Nurolon sutures. One patient developed a pulmonary embolism on Day 2 after surgery. Two patients experienced problems with wound healing, with one of them developing dehiscence and Pseudomonas growth from the wound. One patient experienced a fall after discharge, requiring bur holes for subdural hematoma evacuation. One patient developed an addisonian crisis associated with long-term steroid use. One patient had a gout flare-up in his knee after surgery. Two patients with L1-4 and L2-S1 instrumentation required additional surgeries to extend the constructs due to development of proximal junctional kyphosis. One patient with T9-pelvis instrumentation required a revision surgery due to a dislodged pelvic screw from the rod.

\section{Discussion}

One of the primary benefits of new technology is to make complex processes more accessible to those with less expertise. Spinal fusion surgery involves numerous tasks that the surgeon must complete during a single operation, all with unique pitfalls and risks. As such, it is an ideal procedure in which robotics can be additive. If the robot can automate even one of these procedural steps, this could enhance safety, especially in the hands of a less experi- 


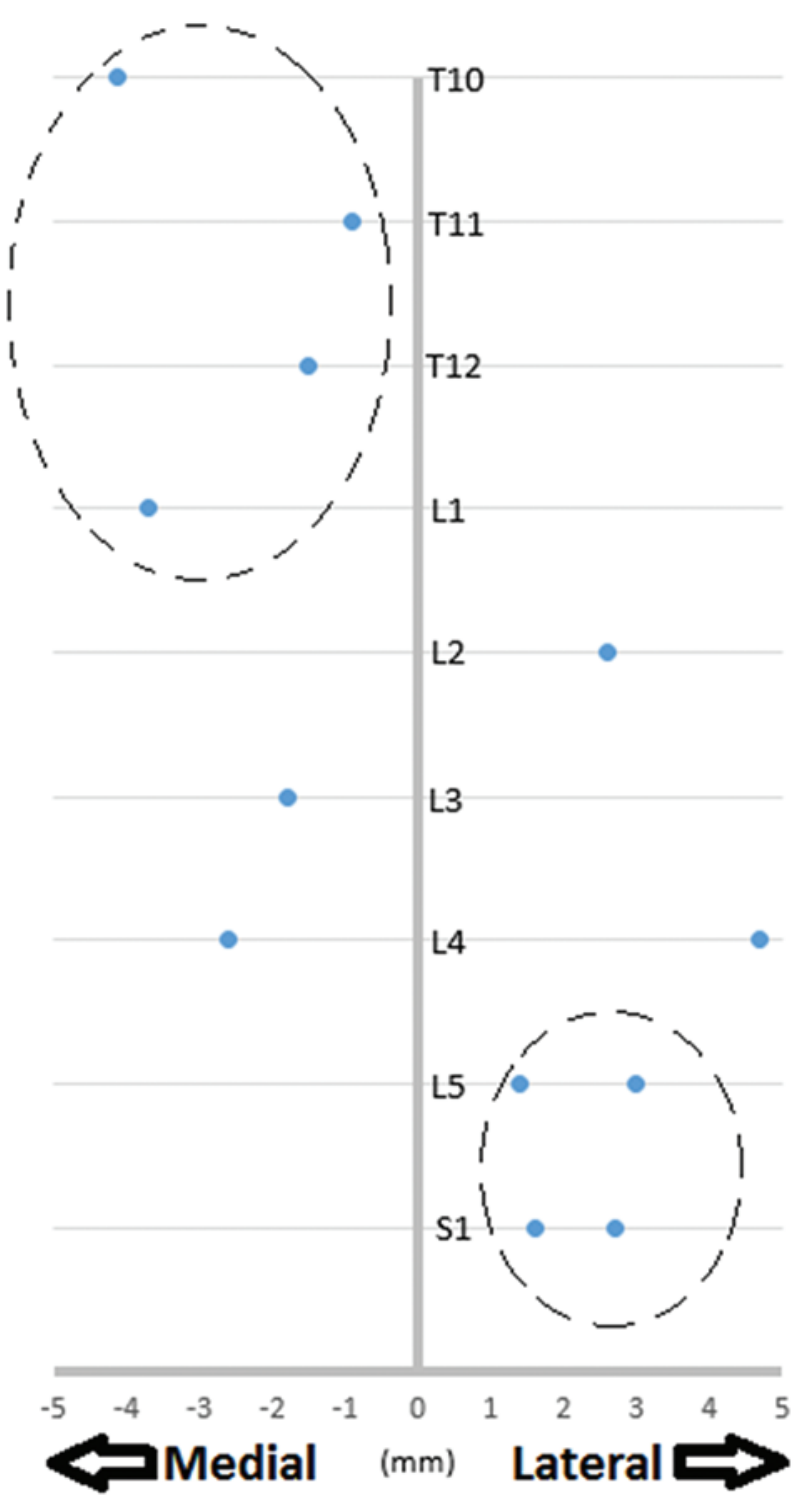

FIG. 5. Shift in the entry point of the breached screws compared with planned trajectories. There were more medially shifted entry points in the lower thoracic area, while there were more laterally shifted points in the lower lumbar area.

enced surgeon. Such a benefit could easily justify its cost as capital equipment.

This study explored this potential benefit using robotics in spinal instrumentation. Since the safe and successful placement of spinal instrumentation is one of the key skill sets of an expert spine surgeon, our research question centered around whether the robot could allow less experienced surgeons to safely place instrumentation under direct supervision and guidance. Previous studies of this robot have all been conducted at specialized centers where the primary robot user was an experienced surgeon who was also committed in philosophy to the use of the robot. Furthermore, if surgeons in training were involved, their numbers were limited. This study was performed at an academic training center with 21 neurosurgical residents and 3 neurosurgery spine fellows. With such a large num- ber of house staff, there was less consistency or familiarity with the robot. As such, this afforded an opportunity to investigate the performance of the robot under unique but real-world conditions, which, to our knowledge, has never previously been explored.

Our study confirmed that the level of experience of the surgeon in training was not associated with a significant advantage when using the robot. Trainees with less than 5 years of experience averaged 4.4 minutes per screw, whereas residents and fellows with 6 or more years of training averaged 4.02 minutes per screw. The difference was not statistically significant $(\mathrm{p}=0.61)$. It should be noted that there was some learning curve associated with robot usage (Fig. 4). This is not surprising, as specific features of robotic surgery, like all procedures, are learned with experience.

We also found that dedication to spine surgery was also not associated with significant differences in screw placement accuracy or efficiency. Among the residents and fellows dedicated to spine surgery the average speed was 3.84 minutes per screw, while nondedicated residents took 4.5 minutes per screw $(\mathrm{p}=0.41)$. This is important, as many neurosurgery residents express an interest in a certain subspecialty and thus will have dedicated a greater amount of their time and energy to acquiring specific subspecialty skills. Thus, it is possible for a senior-level resident who has no interest in spine surgery to actually have less case experience than a much more junior resident who has sought out cases and courses to augment his or her education.

There are many potential implications of this study. As with any assistive technology, its use can lead to enhanced training, but it can also breed dependence. Exploring these effects was not the purpose of this study. However, some of the technical caveats became apparent during the study.

In our early experience, the overall breach rate assessed using postoperative imaging was $11.8 \%$. From the general trend, it appears that the breached thoracic screws favored medial deviation, while breached lower lumbar screws favored lateral deviation in the starting point (Fig. 5). During pedicle screw placement, the initial guide is fixed in space at 2 points. One point is the robotic arm itself and the second point is achieved by tapping the serrated end of the guide into the bone. The robotic arm is not completely rigid and will deviate readily when force is applied, commonly from the tension of the soft tissue. Tapping of the guide into bone may shift the starting point if the tip of the guide skives along the slope of the bone. As a result, thoracic screws can be affected by the anatomy of the transverse process and the lumbar screws by the facet joint capsule. The acute angle of the transverse process in the thoracic spine may be further exaggerated in cases of rotational scoliosis, which adds to the likelihood of the guide sliding medially. The enlarged facet capsule in the lower lumbar spine may provide the opposite effect and push the probe laterally (Fig. 6).

In open cases, the breach can be prevented by leveling the bony surface for the guide or directly visualizing the tip of the guide as it anchors in the bone. This is not possible in percutaneous cases, likely contributing to the explanation of why percutaneous cases appear to have a 

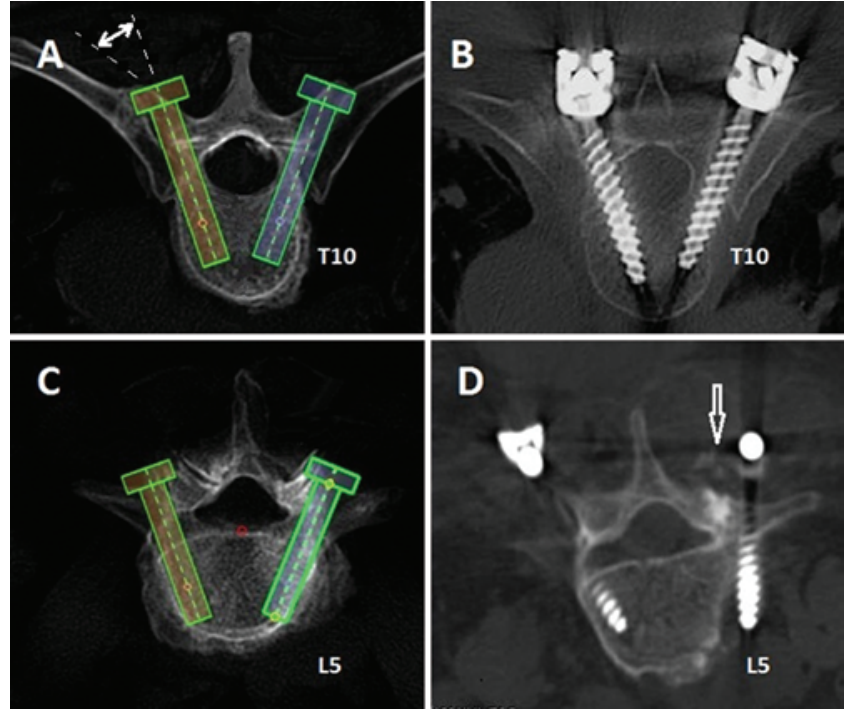

FIG. 6. Thoracic versus lumbar pedicle screw breach propensity. Preoperative trajectory plan (A and $\mathbf{C}$ ) compared with postoperative screw location (B and $\mathbf{D})$. The right thoracic screw is breached medially $(B)$. Note the acute angle of the screw to the transverse process (doubleheaded arrow, A). Left lumbar screw is breached laterally (D). Note the hypertrophied facet capsule (arrow).

higher rate of breach, which was evident in our series as well as in the published literature. ${ }^{2}$

\section{Study Limitations}

This study also has several limitations. The first is the small sample number. Because no training surgeon had more than 7 cases, it is unclear whether the trend toward greater efficiency with more experience and dedication would have reached statistical significance with a larger sample number. However, it is also unclear whether plateau effects would have been achieved with a larger sample number per surgeon in training. Similarly, this study was not a true independent learning curve assessment. That is, all cases were supervised by senior, experienced, and robot-trained surgeons with patient safety as a priority. As such, left without supervision, the results might have been different. However, such a study could not ethically be performed in patients in the United States. It should also be noted that the time per screw represents an estimate based on overall usage of the robot, as detailed earlier. This was standardized in all cases and captured the initiation and conclusion of pedicle screw placement within 2-5 minutes. Finally, this study only explored a single dimension of spine surgery (pedicle screw placement) using a single robot. Whether these findings can be extrapolated to other robotic techniques or other robotic devices is unclear.

\section{Conclusions}

This study offers initial data indicating that robotic assistance with thoracolumbar pedicle screw placement may reduce the skill needed for successful hardware placement. Larger studies investigating the effects of robotic surgery on patient safety, surgeon training, and surgical efficiency will be needed to determine if these findings are generalizable across surgical environments, medical subspecialties, and technology platforms.

\section{References}

1. Han W, Gao ZL, Wang JC, Li YP, Peng X, Rui J, et al: Pedicle screw placement in the thoracic spine: a comparison study of computer-assisted navigation and conventional techniques. Orthopedics 33:10.3928/01477447-20100625-11, 2010

2. Hu X, Ohnmeiss DD, Lieberman IH: Robotic-assisted pedicle screw placement: lessons learned from the first 102 patients. Eur Spine J 22:661-666, 2013

3. Kapoor S, Sharma R, Garg S, Jindal R, Gupta R, Goe A: Navigated pedicle screw placement using computed tomographic data in dorsolumbar fractures. Indian J Orthop 48:555-561, 2014

4. Kim HJ, Kang KT, Park SC, Kwon OH, Son J, Chang BS, et al: Biomechanical advantages of robot-assisted pedicle screw fixation in posterior lumbar interbody fusion compared with freehand technique in a prospective randomized controlled trial-perspective for patient-specific finite element analysis. Spine J [epub ahead of print], 2016

5. Kuo KL, Su YF, Wu CH, Tsai CY, Chang CH, Lin CL, et al: Assessing the intraoperative accuracy of pedicle screw placement by using a bone-mounted miniature robot system through secondary registration. PLoS One 11:e0153235, 2016

6. Lonjon N, Chan-Seng E, Costalat V, Bonnafoux B, Vassal M, Boetto J: Robot-assisted spine surgery: feasibility study through a prospective case-matched analysis. Eur Spine J 25:947-955, 2016

7. Marcus HJ, Cundy TP, Nandi D, Yang GZ, Darzi A: Robotassisted and fluoroscopy-guided pedicle screw placement: a systematic review. Eur Spine J 23:291-297, 2014

8. Schatlo B, Molliqaj G, Cuvinciuc V, Kotowski M, Schaller $\mathrm{K}$, Tessitore E: Safety and accuracy of robot-assisted versus fluoroscopy-guided pedicle screw insertion for degenerative diseases of the lumbar spine: a matched cohort comparison. $\mathbf{J}$ Neurosurg Spine 20:636-643, 2014

9. Tian W: Robot-assisted posterior C1-2 transarticular screw fixation for atlantoaxial instability: a case report. Spine (Phila Pa 2976) 41 (Suppl 19):B2-B5, 2016

\section{Disclosures}

Dr. Wang states that he is a consultant for DePuy Spine, JoiMax, Aesculap Spine, and K2M; is a patent holder with DePuy Spine; and received clinical research support for this study from the Department of Defense.

\section{Author Contributions}

Conception and design: Wang, Urakov. Acquisition of data: Urakov, Chang. Analysis and interpretation of data: Wang, Urakov, Chang. Drafting the article: all authors. Critically revising the article: Wang, Urakov, Burks. Reviewed submitted version of manuscript: all authors. Approved the final version of the manuscript on behalf of all authors: Wang. Statistical analysis: Urakov. Administrative/technical/material support: Burks. Study supervision: Wang.

\section{Correspondence}

Michael Y. Wang, Department of Neurological Surgery, Lois Pope Life Center, 1095 NW 14th Terrace, Miami, FL 33136. email: mwang2@med.miami.edu. 